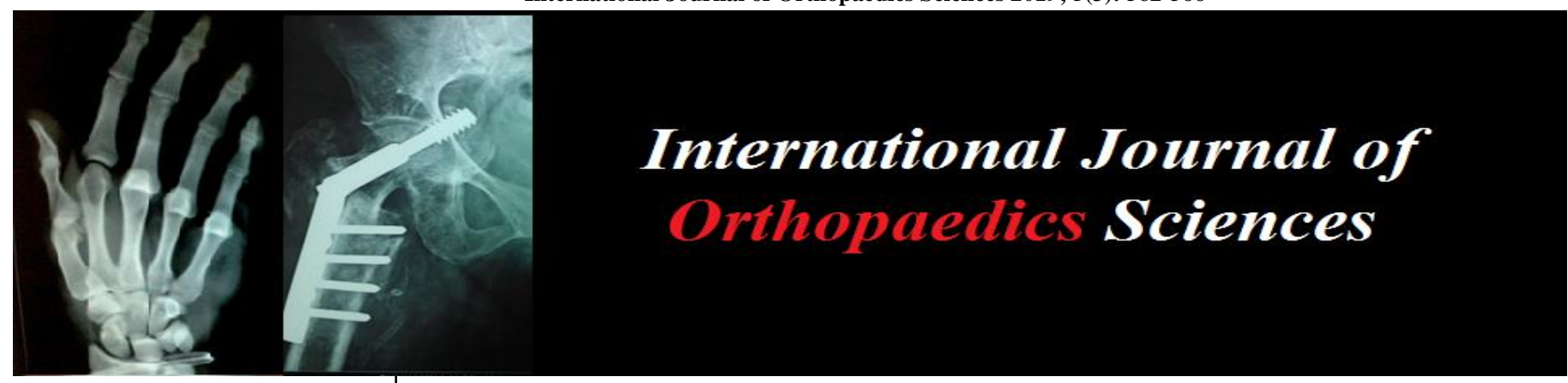

ISSN: $2395-1958$

IJOS 2019; 5(3): 562-566

(C) 2019 IJOS

www.orthopaper.com

Received: 14-05-2019

Accepted: 18-06-2019

Dr. Om Prakash

MS Orthopaedics, Senior

Resident, Mayo Institute of

Medical Sciences, Barabanki,

Lucknow, Utter Pradesh. India

Dr. Lakhan Chandra Pandey MS Orthopaedics, Prof and Head of the Department, Mayo Institute of Medical Sciences,

Barabanki, Lucknow, Utter

Pradesh, India
Correspondence

Dr. Om Prakash

MS Orthopaedics, Senior

Resident, Mayo Institute of

Medical Sciences, Barabanki,

Lucknow, Utter Pradesh, India

\section{Surgical management of spinal tuberculosis via posterior approach: An early outcome}

\author{
Dr. Om Prakash and Dr. Lakhan Chandra Pandey
}

DOI: https://doi.org/10.22271/ortho.2019.v5.i3j.1591

\section{Abstract}

Background: Tuberculosis is a specific infectious disease caused by Mycobacterium Tuberculosis, affects all ages. Spinal tuberculosis is the most common form of skeletal tuberculosis. Treatment of skeletal tuberculosis include antitubercular drugs and surgery was confined to patients failing to respond to drugs or those with complications.

Material and Methods: A prospective study was done from June 2015 to July 2017 including 25 patients with thoracic and lumbar tuberculosis of spine admitted in Ruxmaniben Deepchand Gardi Medical College (RDGMC) Ujjain, fulfilling the inclusion and exclusion criteria. All the patients were treated with antitubercular drugs followed by debridement, decompression and pedicle screw fixation via posterior approach. All the patients were assessed with Frankel grading and Visual analogue scale (VAS). All patients were followed up at monthly interval. Clinical assessment regarding functional outcome was done at the final follow-up.

Result and Conclusion: All the 25 selected patients were assessed clinically, radiologically and on the basis of functional outcome. Excellent result was obtained in 15 cases, Good in 9 cases and Poor in 1 case. All the collected data from this study were analysed statistically.

Keywords: Spinal tuberculosis, thoracic and lumbar spine, debridement, decompression, pedicle screw

\section{Introduction}

Tuberculosis is a specific infectious disease caused by Mycobacterium Tuberculosis ${ }^{[1]}$. The incidence of tuberculosis has been rising since 1980's and 1990's. Extrapulmonary tuberculosis accounts for about $15-20 \%$ of TB cases. Spinal tuberculosis is the most common form of skeletal tuberculosis, constituting approx $50 \%$ of all cases ${ }^{[2]}$. Sir Percival Pott first presented the classical description of spinal tuberculosis in $1779^{[3,4]}$. The evaluation of treatment of TB spine has passed through different phases of development. The development of antitubercular drugs (1948-51), an important milestone divides the treatment of tuberculosis into three eras: 1 . The preantitubercular era, in which the patients were treated either by orthodox, non-operative regimens or by various distant surgical procedures ${ }^{[5]}$. 2 . The postantitubercular era in which all patients were treated operatively in conjunction with antitubercular drugs ${ }^{[6-11]}$. 3. In 1970 Middle Path Regime was followed, in which all patients were treated with antitubercular drugs and surgery was confined to patients failing to respond to drugs or those with complications. Disappointing results of orthodox non operative treatment in the pre chemotherapy era induced surgeons to develop approaches for surgical excision of the diseased bone. Most of the earlier operations were for drainage of abscesses and sinuses, all such procedures resulted in persistent serious sinus and ulcer formation, secondary pyogenic infections, and death in many patients $[12,13,14]$. In post anti-tubercular drugs era spinal tuberculosis was treated with first and second line antitubercular drugs, but the response to absolute non operative treatment showed slow response and its efficacy was doubt full so surgical management was advocated mostly in cases of tubercular paraplegia with the advantage of speedy neural recovery. Several surgical methods were included like anterior approach, anterior decompression combined with posterior stabilization. The combined approach of anterior debridement, interbody fusion combined with posterior instrumentation had some disadvantages like longer operation time, greater blood loss, longer hospitalization and economic burden over patients ${ }^{[15,16]}$. 
In our study, single stage posterior debridement and pedicle screw fixation was done as it has merit over others as duration of hospital stay is significantly reduced and rehabilitation was also faster. Other advantages are reduced morbidity, less blood loss, reduced economic burden to the patient and reduction in surgical site infection.

\section{Material and Methods}

It was a prospective observational study done on 25 patients with thoracic and lumbar tuberculosis of spine, admitted at CRGH hospital associated with R.D. Gardi medical college, Ujjain, fulfilling inclusion and exclusion criteria between June 2015 to July 2017.

\section{Inclusion criteria}

1. Patients of all ages and both sexes.

2. Patients diagnosed clinically, radiologically and on the basis of laboratory findings as case of tuberculosis of spine.

3. Neurological complications which do not show signs of progressive recovery to a satisfactory level after a fair trial of conservative therapy.

4. Patient with spinal caries in whom neurological complications develop during the conservative treatment or become worse while they are undergoing conservative therapy.

5. The Potts spine of thoracic, thoraco-lumbar and lumbar regions.

6. Advanced cases of neurological involvement such as marked sensory and sphincter disturbances or flaccid paralysis.

7. Patient with paravertebral abscess, epidural abscess and intractable pain.

8. Spine disability due to Potts spine.

\section{Exclusion criteria}

1. Patient with other spine disorders.

2. Patients who are medically unfit for surgery.

3. Patient refusing for the procedure.

4. Patients with kyphotic angle more than 60 degree were not included in this study.

All the patients were assessed clinically. A detailed history was obtained and they are subjected to through clinical examination. All patients has symptoms of tuberculosis such as weight loss, evening rise of fever, and fatigue. The findings were noted in proforma. Haematological and radiological investigation were carried out to confirm the diagnosis and to know the level of the lesion. The patient were also assessed preoperatively and postoperatively with the Frankel grading for neurological deficit.

Table: Type of fankel grade

\begin{tabular}{|ll|}
\hline Frankel grade & Definition \\
\hline A & $\begin{array}{c}\text { Complete injury, no motor or sensory function } \\
\text { below the level of injury }\end{array}$ \\
B & Incomplete injury, no motor function \\
C & Incomplete injury, motor function useless, sensory \\
& incomplete \\
D & Incomplete injury, motor function useful, sensory \\
E & incomplete \\
& Incomplete injury, motor function normal, sensory \\
\end{tabular}

All patient underwent decompression and pedicle screw fixation by posterior approach in prone position under general anaesthesia. A midline skin incision was given of $10-15 \mathrm{~cm}$ depending upon the number of levels involved. At the junction of the spinous process and lamina, blunt dissection was performed laterally along the interlaminar space. Dissection was done till the lateral margin of the facet joint was reached. The pedicle screws were inserted under fluoroscopic guidance under the normal vertebrae. The location for screw insertion were identified, at the base of the superior articular facet joint and the midline of transverse process. On one side temporary rod, that had been contoured to accommodate the deformity, was applied to maintain spinal stability and to avoid any cord injury during decompression and debridement. At the thoracic level costotransversectomy was done. Paravertebral and epidural abscess was drained and through debridement of sequestra and necrotic debris was done until the fresh bleeding was seen and decompression of cord was done. The same procedure was carried out on the opposite side if required. Tissues from the diseased area was harvested for the histopathological confirmation of disease. Opposite side contour rod was fixed and carefully gradual correction of kyphosis was done with the help of the compression and streching of the internal fixation instrumentation.

In post-operative care dressing was done on $2^{\text {nd }}$ post-operative day. Bed rest was recommended for all patients and all were provided with nutritional and supportive therapy. After one week, patients were encouraged to stand or walk with effective support of braces. Follow up care consisting of physical and radiological examination, ESR and CRP values, correction of deformity and assessment of recovery of neurological function by Frankel grading. Long journeys were avoided for 3 months. Lifting weight and bending was prohibited.

\section{Observation and Result}

Twenty five patients of thoracic and lumbar tuberculosis of spine that deamed fit into inlusion criteria were selected as subject for the present study. All the patients were subjected to debridement, decompression and pedicle screw fixation via posterior approach.

The age of the patients ranges from 18 to 65 years with an average of 37.8 years. 8 patients were below the age of 30 years, 9 patients between 30-40 years and 8 above 40 years. Male patients were predominant in our study with 20 males $(80 \%)$ and 5 female $(20 \%)$ patients out of 25. D12-L1 level was most commonly involved. The mean kyphotic angle preoperatively in our study was 18.88 degree which decreased significantly to an average of 4.96 degree postoperatively. Most of the patients were in grade D according to the Frankel grading system. Ten patients $(40 \%)$ out of 25 were in grade $\mathrm{D}, 7(28 \%)$ in grade $\mathrm{E}, 6(24 \%)$ were in grade $\mathrm{C}$ and 2 patients $(8 \%)$ were in grade B pre operatively. Postoperatively 20 patients $(80 \%)$ were in grade E, $4(16 \%)$ improved to grade D and $1(4 \%)$ was in grade $C$.

\section{Functional outcome}

The functional outcome was assessed on the basis of postoperative Frankel grading, VAS scoring, kyphotic angle, ESR and CRP levels. Preoperatively only 7 patients were in grade $\mathrm{E}$ according to Frankel grading, which increased to 20 patients postoperatively. The mean VAS score preoperatively was 6.76 which dropped to 0.88 postoperatively. The preoperative mean ESR and CRP was $37.24 \mathrm{~mm} / \mathrm{hr}$ and $14.28 \mathrm{mg} / \mathrm{L}$ respectively, which dropped to an average of 
$25.12 \mathrm{~mm} / \mathrm{hr}$ ESR and $9.79 \mathrm{mg} / \mathrm{L}$ CRP postoperatively. The follow up of the patients was done at 3 months, 6 months, 9 months and 12 months. The surgical outcome in 15 patients $(60 \%)$ was excellent, 9 patient $(36 \%)$ had good result and 1 (4\%) patient had poor result. In 2 patients surgical site infection was seen and 1 patient had implant failure. No neurological deterioration was found postoperatively.

Table 1: Age distribution

\begin{tabular}{|c|c|c|}
\hline Age & No. of Cases & \% \\
\hline$<=30$ & 8 & 32.0 \\
\hline $31-40$ & 9 & 36.0 \\
\hline$>40$ & 8 & 32.0 \\
\hline Total & 25 & 100 \\
\hline
\end{tabular}

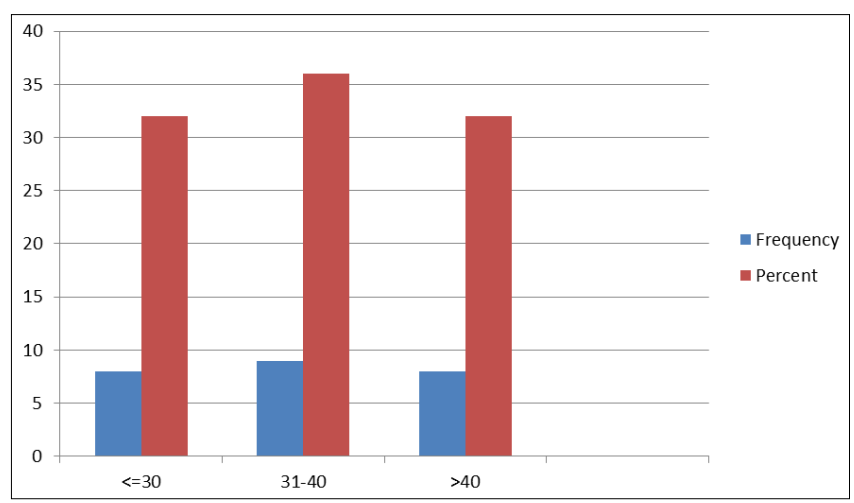

Graph 1: Age distribution

Table 2: Sex Distribution

\begin{tabular}{|c|c|c|}
\hline Sex & No. of Cases & \% \\
\hline Female & 5 & 20.0 \\
\hline Male & 20 & 80.0 \\
\hline Total & 25 & 100 \\
\hline
\end{tabular}

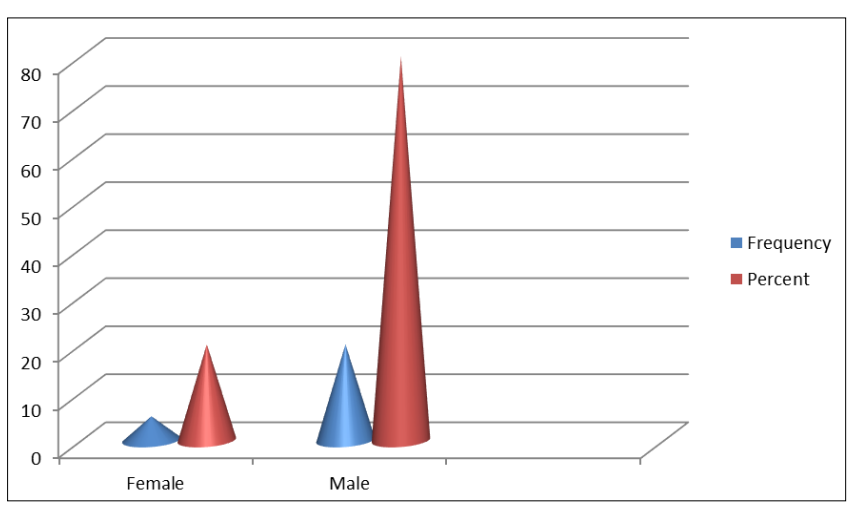

Graph 2: Sex Distribution

Table 3: Distribution of Level involved

\begin{tabular}{|c|c|c|}
\hline Levels involved & No. of Cases & \% \\
\hline D9-D10 & 1 & 4.0 \\
\hline D10-D11 & 3 & 12.0 \\
\hline D10-D12 & 2 & 8.0 \\
\hline D11-L1 & 4 & 16.0 \\
\hline D12-L1 & 5 & 20.0 \\
\hline D12-L5 & 1 & 4.0 \\
\hline L1-L2 & 2 & 8.0 \\
\hline L3-L4 & 3 & 12.0 \\
\hline L4-L5 & 2 & 8.0 \\
\hline L5-S1 & 2 & 8.0 \\
\hline Total & 25 & 100 \\
\hline
\end{tabular}

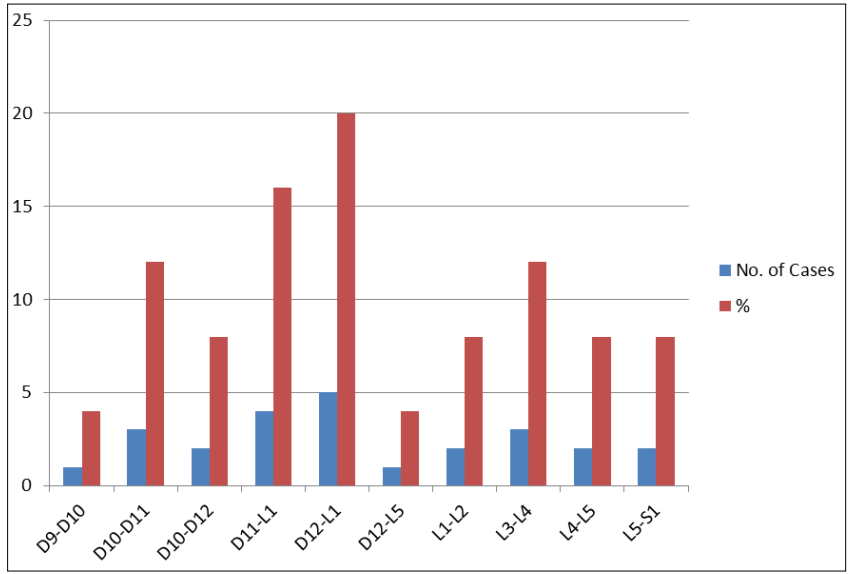

Graph 3: Distribution of Level involved

Table 4: Distribution of frankel grade preoperatively

\begin{tabular}{|c|c|c|}
\hline Frankel Grade & No. of Cases & $\boldsymbol{\%}$ \\
\hline A & 0 & 0.0 \\
\hline B & 2 & 8.0 \\
\hline C & 6 & 24.0 \\
\hline D & 10 & 40.0 \\
\hline E & 7 & 28.0 \\
\hline Total & 25 & 100 \\
\hline
\end{tabular}

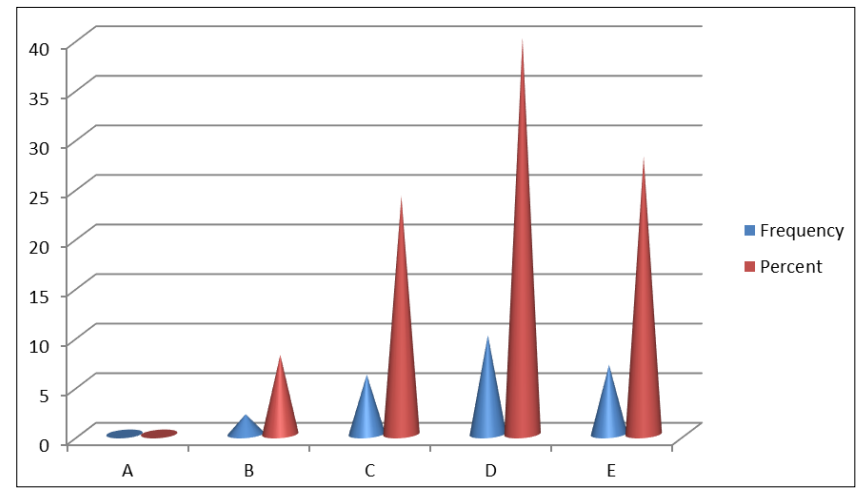

Graph 4: Distribution of frankel grade preoperatively

Table 5: Distribution of frankel grade post-operatively

\begin{tabular}{|c|c|c|}
\hline Frankel Grade & No. of Cases & \% \\
\hline A & 0 & 0.0 \\
\hline B & 0 & 0.0 \\
\hline C & 1 & 4.0 \\
\hline D & 4 & 16.0 \\
\hline E & 20 & 80.0 \\
\hline Total & 25 & 100 \\
\hline
\end{tabular}

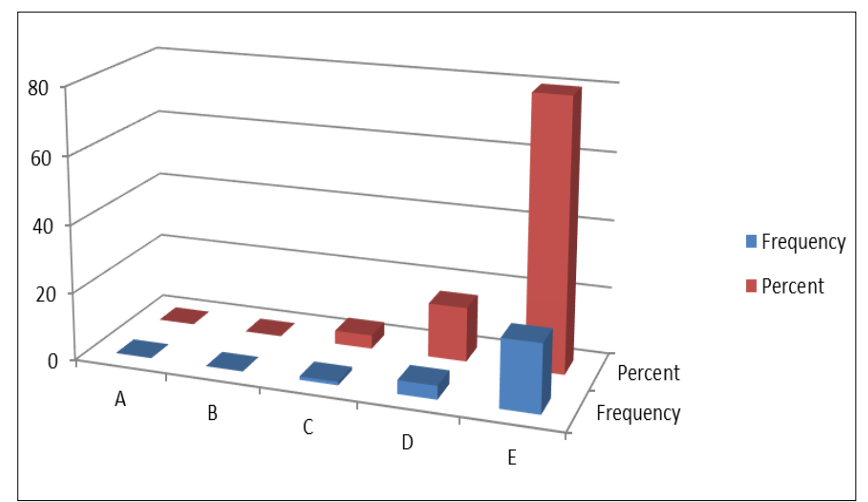

Graph 5: Distribution of Frankel Grade Post-operatively 
Table 6: Distribution of Surgical Outcome

\begin{tabular}{|c|c|c|}
\hline Outcome & No. of Cases & \% \\
\hline Excellent & 15 & 60.0 \\
\hline Good & 9 & 36.0 \\
\hline Poor & 1 & 4.0 \\
\hline Total & 25 & 100 \\
\hline
\end{tabular}

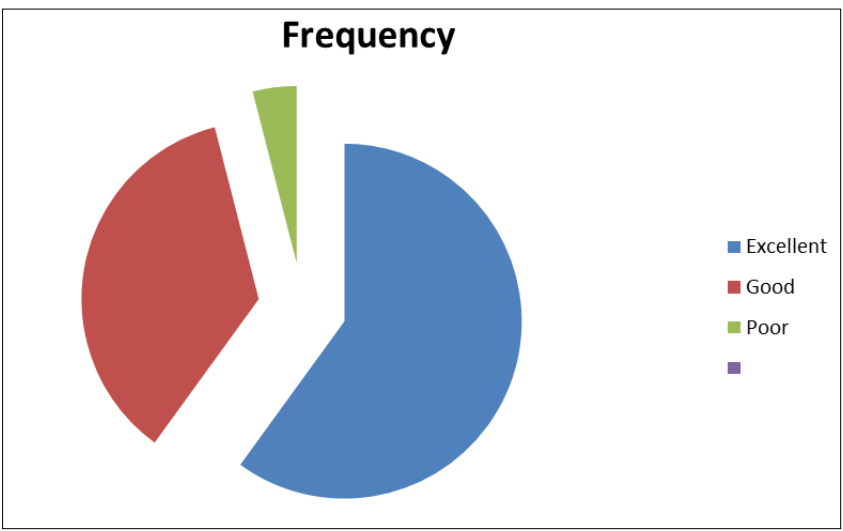

Graph 6: Distribution of Surgical Outcome

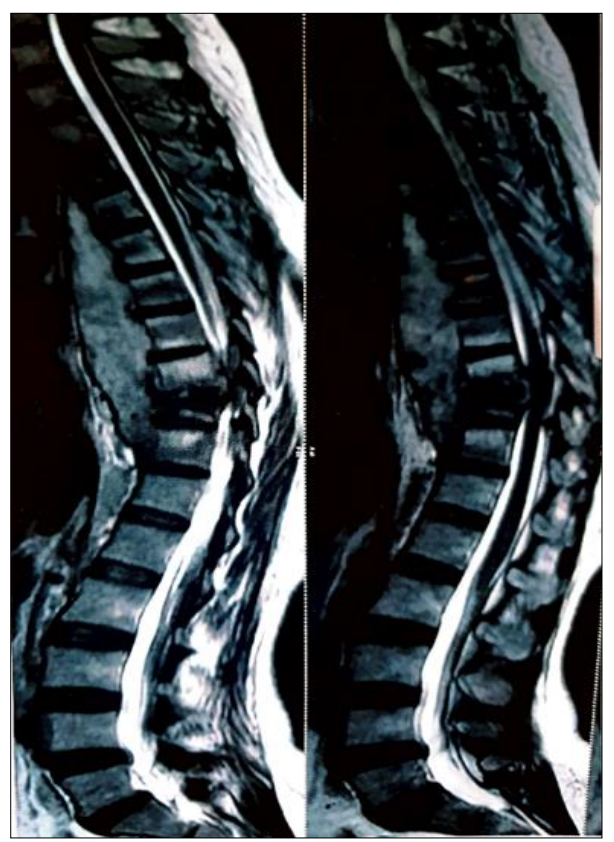

Fig 1: Preoperative MRI involving dorsal spine

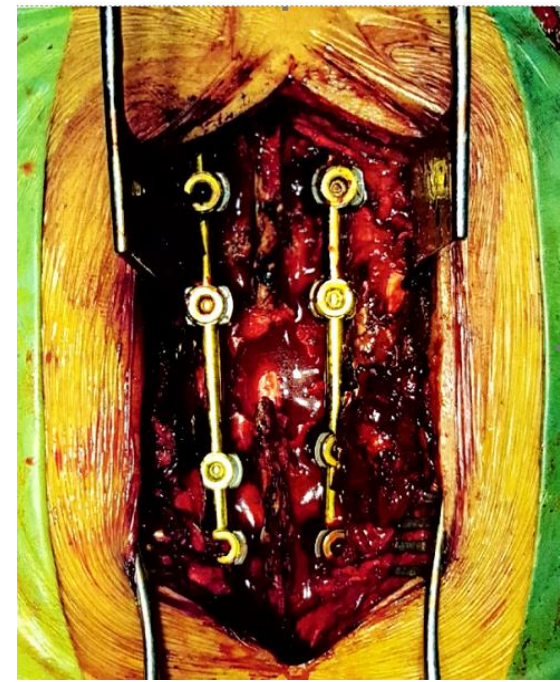

Fig 2: Operative image showing Pedicle screw and Rod in position

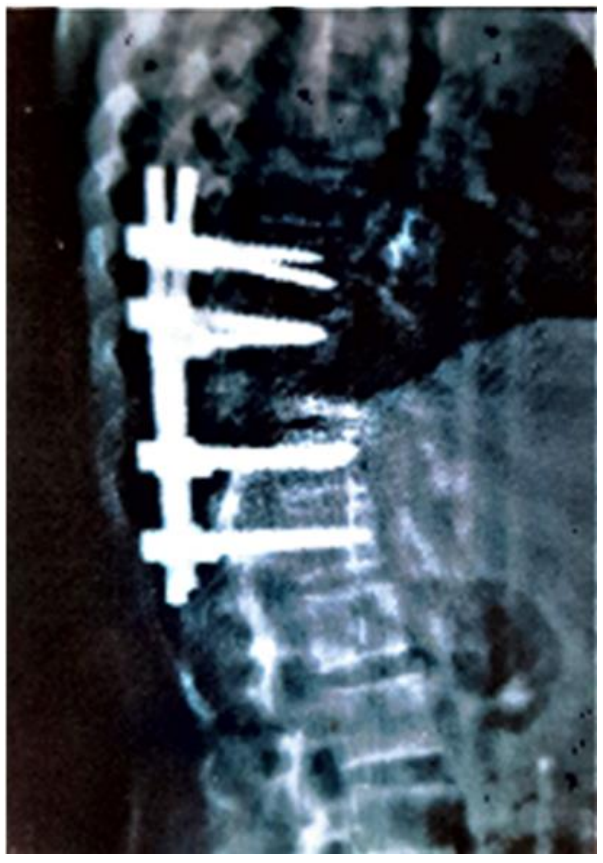

Fig 3: Post-operative X-ray showing implant in position

\section{Discussion}

Spinal tuberculosis has been a global problem since ancient times and estimated that approx 6 billion people are infected with tuberculosis and over 9 million cases of active tuberculosis occur annually with 2-3 million deaths. The incidene of tuberculosis has increased recently because of large scale migration, emergence of antibiotic resistance strain and other reasons. As we are one of the developing country the disease is associated with poor socio economic status. WHO suggested that the human immunodeficiency virus pandemic is a risk factor for acquired tuberculosis and had little impact on epidemiology of spinal tuberculosis, although a large French study, none of the 82 cases of spinal tuberculosis were HIV infected same observation in our study $[17,18]$

Several surgical approaches have been introduced including anterior spinal fusion, anterior-posterior spinal fusion, posterior spinal fusion alone and combined approach. Tuberculosis of spine almost always affect the anterior column of spine and hence anterior approach is considered the gold standard for debridement and decompression in Potts spine which was popularized by Hodgson ${ }^{[19]}$.

Zhang et al. reported 1 stage posterior debridement and instrumentation for treating thoracic and lumbar tuberculosis of spine, the grafted bone was fused within 10 month in all patients and the kyphotic angle was significantly corrected ${ }^{[20]}$. The posterior approach has some advantages over the surgical procedures, the operative procedures of debridement, spinal cord decompression, deformity correction, bone grafting and internal fixation can also be completed via only one incision and in one position with better correction of deformity than anterior approach and also having fewer postoperative complications ${ }^{[20]}$.

Md. Shah Alam et al. did study over 582 patients of spinal tuberculosis out of which 113 cases were treated with thoracotomy along with anterolateral decompression and autogenus strut bone grafting with simultaneous fixation by screws and rods, and in remaining 449 patients posterior decompression, posterior interbody and posterolateral fusion by bone graft with stabilization by transpedicular screws and rods were done, Revision surgery was performed in 6 
patients, implant failure occurred in 4 patients while malposition of screws occurred in 12 patients, Neurological improvement occurred in all patients except in 2 patients ${ }^{[17]}$.

The advent of pedicle screw instrumentation at the development of the extended posterior approaches have allowed the surgeon to perform anterior debridement and reconstruction from behind and have led many surgeon to opt for posterior only surgery ${ }^{[20]}$.

Hongqi zhang et al. carried out a retrospective study of spinal tuberculosis of 28 patient treated surgically by debridement, internal fixation and reconstruction with specially formed titanium mesh cage via posterior only approach, the VAS score dropped from preoperative level of 6.31 to the final followup level of 0.57 postoperatively ${ }^{[21]}$.

Anil kumar Jain et al. analyzed all articles in which instrumented stabilization was reported over the last 20 years [22]. There were 1097 patients stabilized by either anterior or posterior instrumentation in 123 analyzed series. In anterior instrumentation, mean preoperative kyphotic angle was 25.35 degree, immediate postoperative kyphotic angle was 9.8 degrees and final kyphosis was 12.97 degrees. In posterior instrumentation, mean kyphosis correction achieved was 19.03 degrees with initial kyphosis was 30-35 degree which reduced to $15-18$ degrees ${ }^{[22]}$

Though the anterior approach is favoured method for surgical management of tuberculosis of spine, as the lesion is situated anteriorly. The debridement, decompression and posterior instrumentation is an effective and safe method. The rate of morbidity is less, lesser complications, the duration of surgery and blood loss is also less in comparision to other approaches. In our study we have also observed favourable results with posterior approach. According to Frankel grading there was improvement in neurological deficit. The kyphotic angle reduced from mean 18.88 properatively to 4.96 postoperatively. There was marked reduction in preoperative ESR and CRP level post-operatively.

\section{Conclusion}

From our study we conclude that the debridement, decompression and transpedicular screw fixation is safe and effective method for treating tuberculosis of spine via single posterior only approach with advantage of less operation time, good neurological recovery, less postoperative complications and adequate correction of kyphotic deformity. This study have certain limitation in term of small sample size, shorter duration of follow up and authors experience.

\section{References}

1. Tuli SM. tuberculosis of the spine: a historical review. Clin Orthop Relat Res. 2007; 460:29-38.

2. Tuli SM. general principle of osteoarticular tuberculosis. Clin ortho Relat Res, 2002, 11-19.

3. Gordon, Richard. The alarming history of medicine. New York: St Martin's press. ISBN 0-312-10411-1, 1994.

4. Dobson J, Percival Pott in annals of the royal college of surgeons of England. 1972; 50(1972):54-56.

5. Bick KM. classics of orthopaedics. Philadelphia: JB Lippincott co, 1976.

6. Cameron JA, Robinson CL, Robertson DE. The radical treatment of Pott's disease and Pott's paraplegia by extirpation of the diseased area and anterior spinal fusion. Am Rev Respir Dis. 1962; 86:76-80.

7. Hodgson AR, Stock FE, Fang HS, Ong GB. Anterior spinal fusion. The operative approach and pathological findings in 412 patients with Pott's disease of the spine. Br J Surg. 1960; 48:172-178.

8. Chahal AS, Jyoti SP. The radical treatment of tuberculosis of the spine. Int Orthop. 1980; 4(2):93-9.

9. Mukhopadhaya B, Mishra NK. Tuberculosis of spine. Indian J Surg. 1957; 19:59-81.

10. Fellander M. Radical operation in tuberculosis of the spine. Acta orthop scand suppl. 1955; 19:1-117.

11. Kohli SB. Radical surgical approach to spinal tuberculosis. J Bone Joint Surg Br. 1967; 49:668-673.

12. Dobson J. Tuberculosis of spine; an analysis of the result of conservative treatment and of the factors influencing the prognosis. J Bone Surg Br. 1951; 33-B(4):517-531.

13. Seddon HJ. Potts paraplegia, prognosis and treatment. $\mathrm{Br}$ J Surg. 1935; 22:769-799. DOI: 10.1002/BJS.1800228813.

14. Mercer W. then and now: the history of skeletal tuberculosis. Jr Coll Surg Edinb. 1964; 9:243-254.

15. Zhang H-Q et al. One-stage posterior debridement, transforaminal lumbar interbody fusion and instrumentation in treatment of lumbar spinal tuberculosis: a retrospective case series. Archives of orthopaedic and trauma surgery. 2013; 133:333-341. 10.1007/s00402-012-1669-2

16. Zhang HQ et al. Surgical management by one-stage posterior transforaminal lumbar debridement, interbody fusion, and posterior instrumentation for lumbo-sacral tuberculosis in the aged. Arch Orthop Trauma Surg. 2012; 132:1677-1683. DOI: 10.1007/s00402-0121604-6.

17. Md. Shah Alam et al. Surgery for spinal tuberculosis: a multi-center experience of 582 casesJ Spine Surg. 2015; 1(1):65-71. DOI: 10.3978/j.issn.2414-469X.2015.07.03

18. Jairam Jagiasi1 D, Mihir Patel R. Results of management of spinal tuberculosis according to middle path regime and short course chemotheraphy. Jagiasi JD et al. Int $\mathbf{J}$ Res Orthop. 2017; 3(5):966-972.

19. Hodgson AR et al. Anterior spinal fusion. The operative approach and pathological findings in 412 patients with Pott's disease of the spine. Br J Surg. 1960; 48:172-8.

20. Rajasekaran S. Kyphotic deformity in spinal tuberculosis and its management. Int Orthop. 2012; 36:359-65.

21. Hongqi Zhang email author, kefeng zeng et al. debridement, internal fixation, and reconstruction using titaniu mesh for the surgical treatment of thoracic and lumbar spinal tuberculosis via a posterior only approach: a 4 year follow up of 28 patients. jpournal of orthopaedic surgery and research. December. 2015; 10:150.

22. Anil Kumar Jain, Saurabh Jain. Instrumented stabilization in spinal tuberculosis Int Orthop. 2012; 36(2):285-292. Published online 2011 Jul1. DOI: 10.1007/s00264-011-1296-5. 